\title{
Leaky Coaxial Cable with Continuous Scanning Directive Beam
}

\author{
Ahmed Radwan $^{(1)}$,Zeeshen Siddiqui( ${ }^{(1)}$, Marko Sonkki ${ }^{(1)}$, Marko Tuhkala(2), Sami Myllymäki ${ }^{(2)}$ \\ (1) Centre for Wireless Communications (CWC), University of Oulu, Finland. \\ ${ }^{(2)}$ Microelectronics and Materials Physics laboratories, University of Oulu, Finland.
}

\begin{abstract}
Leaky Coaxial Cable (LCX) is an antenna array used for many applications such as train communication, highway information broadcasting and object detection sensors. We propose leaky Coaxial cable which it can continuously scan a directive beam over a wide range of angles by just varying the frequency with improved radiation efficiency. Furthermore, we can obtain a continuous directive beams over the top and the bottom face of the LCX by adding slots array at both sides.
\end{abstract}

Key words- Antenna, Distributed Antenna System, Leaky Coaxial Cable.

\section{INTRODUCTION}

Leaky feeders are special form of normal coaxial cables where the outer shield has a series of slots or holes with different geometries and spacing between them [1], and so it acts as both a transmission line and an antenna. Leaky feeders can be considered as distributed antenna which waves propagate through the dielectric and currents running along the inner and outer coaxial conductors.

The development of the leaky coaxial cables is moving toward high frequencies and wide bandwidths [2-3] to provide communication links in many places, such as subways, underground, shops, and generally indoor environment. The applications have a wide range from mobile phone to WiFi and Wlan systems. They have recently been considered as an alternative for conventional antenna for indoor applications as well [4]. Leakywave radiations are usually generated by leaking the electromagnetic energy gradually over a structure. The radiated beams can be scanned in the space as frequency changes in operation or the beam can be designed to radiate in the direction under interest in a certain frequency.

\section{ANTENNA DESIGN AND SIMULATION}

Leaky coaxial cable is one kind of leaky waveguide with periodic slots on its outer conductor. It is composed of following five parts; inner and outer conductors can be viewed as the perfect conductors, insulating jacket, slots, and the dielectric layer between the conductors which in our design has a relative permittivity of 1.2.

Usually, the design procedure for the leaky coaxial cables with periodic slots includes two steps; the design for the desired frequency band and the design to meet the coupling loss requirements. Because the frequency band of the cable depends mainly on the arrangement of the slots, it is usually studied first. A combination of slot arrays and an axially continues slots is proposed here for a beam scanning LCX.

Based on [4], starting from the design of a single slot spaced by a gab $\mathrm{P}_{1}$ to the other slot, and when multiple slots are combined, they behave as an array. $\mathrm{P}$ is the period of slots, which it can be obtained by using the following formula [2]:

$$
\lambda /\left(\sqrt{ } \varepsilon_{\mathrm{r}}+1\right)<\mathrm{P}<2 \lambda /\left(\sqrt{ } \varepsilon_{\mathrm{r}}+1\right)
$$


as $\varepsilon_{\mathrm{r}}$ is the relative permittivity of dielectric material between the conductors and the operation band is bounded by $f_{1}$ and $f_{2}$ as $-\mathrm{m} f_{1}<f_{0}<-\mathrm{m} f_{2}$ where $f_{1}=\mathrm{c} /\left[\mathrm{P}\left(\sqrt{ } \varepsilon_{\mathrm{r}}+1\right)\right], f_{2}=\mathrm{c} /\left[\mathrm{P}\left(\sqrt{ } \varepsilon_{\mathrm{r}}-1\right)\right], \mathrm{P} 1=\mathrm{P} / 4$ and c is the velocity of light, with m equal to -1 to suppress the harmonic radiation from the proposed LCX as in [4]. In this paper, the simulations of this cable are performed by the electromagnetic simulator CST [5]. The configuration of the proposed LCX is shown in Fig. 1 with specified dimensions where $\left(r_{1}\right.$ is the radius of the inner conductor and equals to $7 \mathrm{~mm}, \mathrm{r}_{2}$ is the radius of the outer conductor and equals to $12.5 \mathrm{~mm}, \mathrm{P}_{1}$ is the opening part between the two slots which is equal to $7 \mathrm{~mm}, \mathrm{P}=100$, The width and length of the slots are $\mathrm{a}=3.75 \mathrm{~mm}$, and $\mathrm{b}=2 \mathrm{~mm}$ respectively, and the full length of the cable is $2 \mathrm{~m}$.

The radiation patterns of the proposed leaky cable are shown in Fig. 2. The figures demonstrate the continuous directive beam over a wide range of frequencies from $1.1 \mathrm{GHz}$ to $2.9 \mathrm{GHz}$ with a center frequency at $1.9 \mathrm{GHz}$. The beam steering is presented from $30^{\circ}$ to $110^{\circ}$ over the entire frequency band and also with improved leakage radiation energy and gains. This operating band of frequencies can be controlled by many parameters such as the distance between the slots (P) and the width of the continues axially slot (b).

In case of using the same proposed design over both sides of the LCX, we can obtain the same freedom of controlling the beams over the same frequency band but it will be on both sides, as in some applications need to have radiation pattern in both sides of the cable. The radiation patterns are shown in Fig. 3 with the same beam scanning angles as in Fig. 2. Based on the above results, a leaky feeder cable can support optimal coverage and reduces the occurrence of the leaky cable radiating in undesirable directions or locations along its installed path.
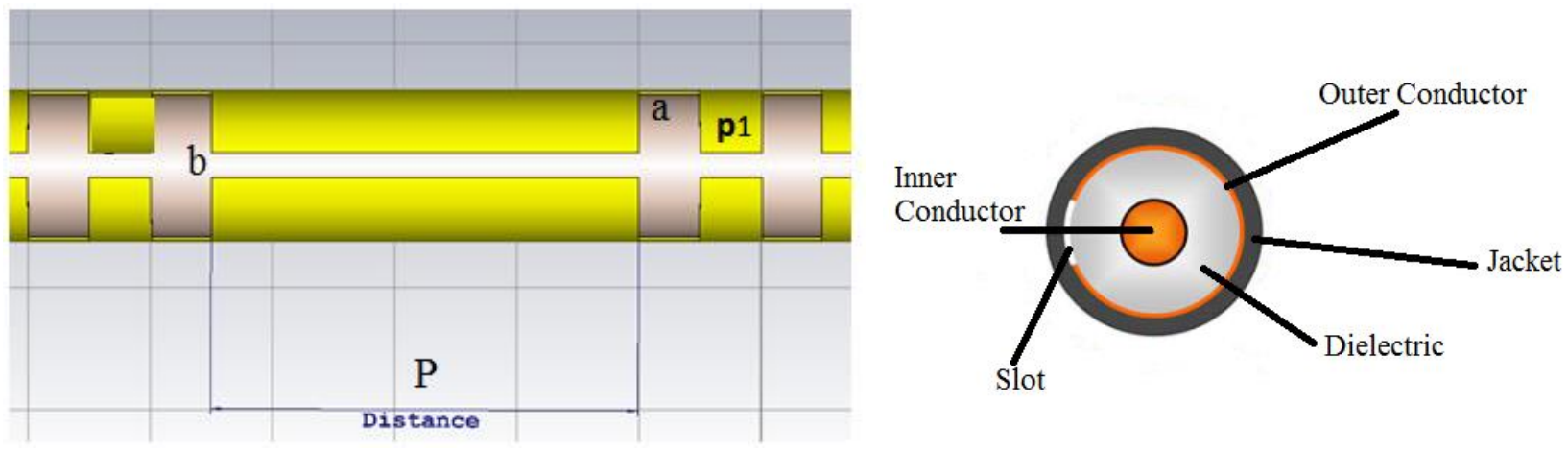

Fig.1 The proposed leaky coaxial cable with slots on one side of the cable.

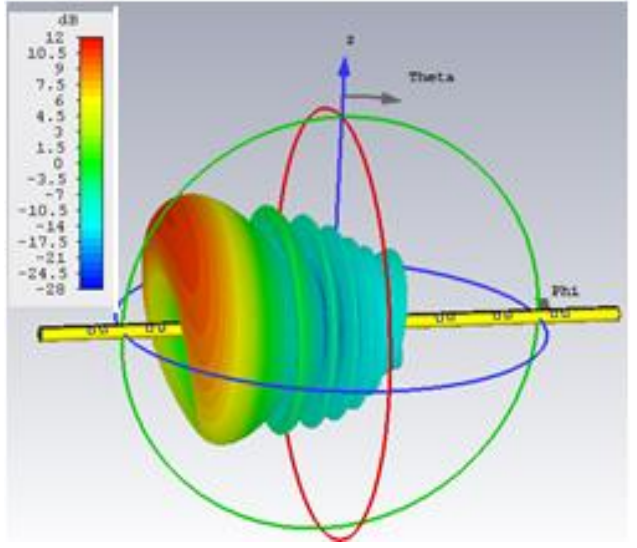

$1.1 \mathrm{GHz}$

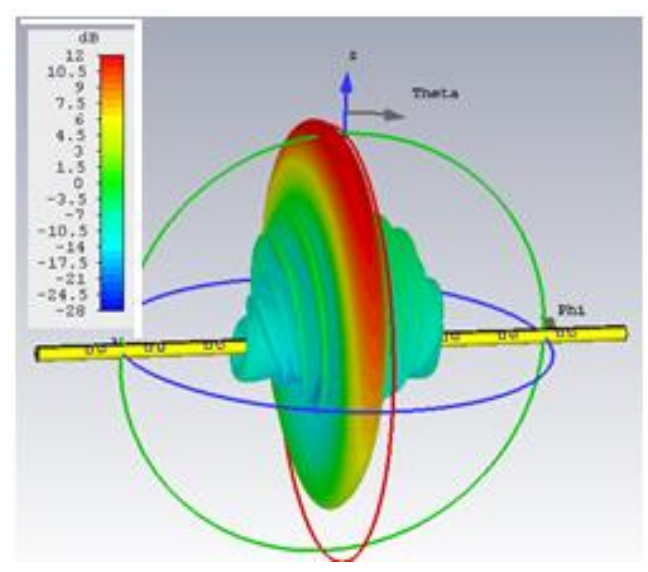

$1.9 \mathrm{GHz}$

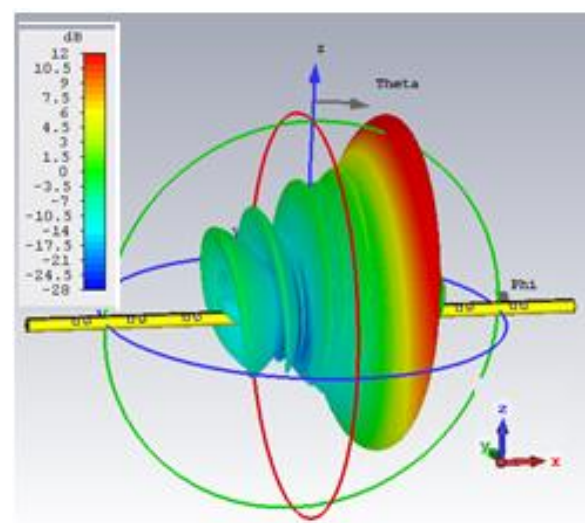

$2.9 \mathrm{GHz}$

Fig.2 Radiation pattern of the cable with slots on one side. 


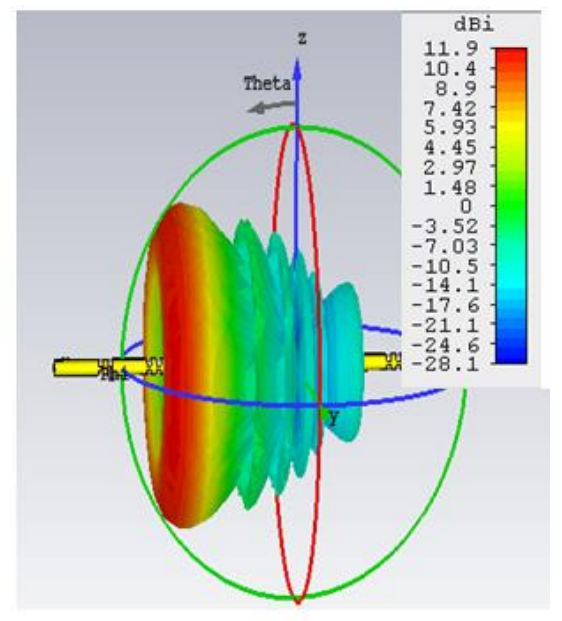

$1.1 \mathrm{GHz}$

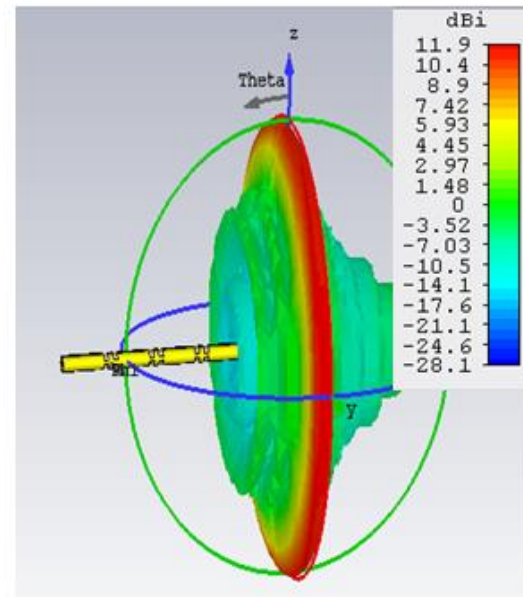

$1.9 \mathrm{GHz}$

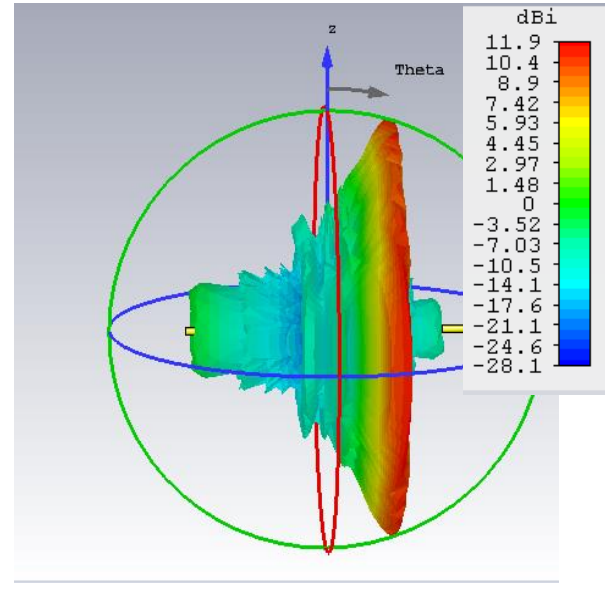

$2.9 \mathrm{GHz}$

Fig.3 Radiation pattern of double sided LCX

\section{CONCLUSIONS}

In this paper, we proposed a Leaky Coaxial Cable with continuous axially slot added to an array of slots following a set of design procedures. By using such a design, the leaky cable can continuously scan a directive beam over a wide range of angles by just varying the frequency. The proposed structure also presented high radiation efficiency over the studied wide operation band.

\section{ACKNOWLEDGEMENT}

The work has received funding from Finnish Funding Agency for Innovation, Tekes regarding Smart Wireless Cable -Project. We are thankful to our sponsors and the industrial collaborators, Prysmian Group, Elcoflex and Premix.

\section{REFERENCES}

[1] Farahneh, H. and Fernando, X., 2014, May. Modeling the leaky feeder as a multi antenna array. In Electrical and Computer Engineering (CCECE), 2014 IEEE 27th Canadian Conference on (pp. 1-5).

[2] Wang, J.H.and Mei, K.K., 2001. Theory and analysis of leaky coaxial cables with periodic slots. IEEE transactions on antennas and propagation, 49(12), pp.1723-1732.

[3] P. P.Delogne and L.Deryck, “Underground use of a coaxial cable with leaky sections,"IEEE Trans. Antennas Propagat., vol. AP-28, pp.875-883, Nov. 1980.

[4] Wang, J.H. and Mei, K.K., 2001. Design and calculation of the directional leaky coaxial cables. Radio Science, 36(4), pp.551-558.

[5] https://www.cst.com/ 